\title{
The molecular basis of NOD2 susceptibility mutations in Crohn's disease
}

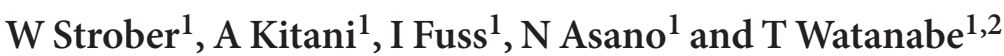

Nucleotide oligomerization domain (NOD)2 is a member of the NOD-like receptor family of proteins that initiate inflammatory responses when exposed to ligands derived from bacterial components that gain access to the intracellular milieu. It is thus somewhat paradoxical that polymorphisms in the gene that encode NOD2 (CARD15) that lead to impaired NOD2 function, are susceptibility factors in Crohn's disease, a condition marked by excessive inflammatory responses to normal bacterial flora. In an initial series of studies conducted in our laboratory to better define NOD2 function and to resolve this paradox we showed that NOD2 activation by its ligand, muramyl dipeptide (MDP) ordinarily downregulates responses to Toll-like receptor (TLR) stimulation, and thus cells lacking NOD2 mount increased responses to such stimulation. This fits with the fact that mice bearing an NOD2 transgene, and thus having cells with increased NOD2 function display decreased responses to TLR stimulation and are resistant to experimental colitis induction. In further studies, we showed that prestimulation of cells with NOD2 ligand renders them unresponsive to TLR stimulation, because such prestimulation results in the elaboration of inhibitory factor (IRF4), an inhibitor of TLR-induced inflammatory pathways. Furthermore, administration of MDP to normal mice induces IRF4 and prevents experimental colitis. These studies strongly suggest that NOD2 polymorphisms are associated with Crohn's disease because they lead to a decrease in the negative regulation of TLR responses occurring in the normal gut, and thus a pathologic increase in responses to the normal flora. The finding that MDP administration prevents experimental colitis opens the door to the possibility that such treatment might quell Crohn's disease relapses in patients without NOD2 abnormalities.

\section{INTRODUCTION}

The discovery a half decade ago that some $15 \%$ of patients with Crohn's disease bear a homozygous or compound heterozygous mutation in the gene that encodes nucleotide oligomerization domain (NOD)2 (the CARD15 gene) has opened a new and unquestionably important window on the pathogenesis of this disease. ${ }^{1-3}$ If we can understand how this mutation creates susceptibility in some patients with Crohn's disease we can establish an invaluable paradigm for the causation of disease in all patients.

NOD2 is an intracytoplasmic member of the family of proteins now known as the NOD-like receptor proteins. ${ }^{1-4}$ These proteins are usually composed of a central NOD flanked on its $C$-terminal side by a leucine-rich repeat domain and on its $N$-terminal side by a CARD or pyrin domain. The leucine-rich repeat domain recognizes microbial components and the CARD or pyrin domain interacts with downstream molecules to bring about effector function. NOD2 has been shown to recognize muramyl dipeptide (MDP), a component of peptidoglycan, itself a component of the bacterial wall of virtually all bacteria.

Upon interaction with its ligand, MDP, NOD2 undergoes a conformational change that allows it to interact with a downstream adaptor molecule known as receptor interacting caspaselike kinase (RIP2). Receptor interacting caspase-like kinase, in turn, induces the polyubiquitination of NF- $\kappa \mathrm{B}$ essential modulator $(\mathrm{I} \kappa \kappa \gamma)$ the key scaffolding protein of $\mathrm{NF}-\kappa \mathrm{B}$ and thus initiates NF- $\kappa \mathrm{B}$ activation and its downstream panoply of inflammatory cytokines, including IL-12. ${ }^{4}$ The ability of NOD2 to recognize a more or less ubiquitous bacterial component positions this molecule to mediate an inflammatory response and thus play a role in the induction of Crohn's inflammation. Thus, if a mutation in NOD2 results in loss of the ability of NOD2 to

\footnotetext{
${ }^{1}$ Mucosal Immunity Section, Laboratory of Host Defenses, NIAID, NIH, Bethesda, Maryland, USA. ²Department of Gastroenterology and Hepatology, Kyoto University
} Graduate School of Medicine, Kyoto, Japan. Correspondence: W Strober (wstrober@niaid.nih.gov) 
activate NF- $\kappa \mathrm{B}$, (as has indeed been shown) then the mutation would be expected to lead to decreased NF- $\kappa \mathrm{B}$ activation and decreased inflammation rather than increased $\mathrm{NF}-\kappa \mathrm{B}$ activation and inflammation that in fact characterizes Crohn's disease.

\section{NOD2 REGULATORY FUNCTION}

A possible solution to this conundrum was provided several years ago with our demonstration that antigen-presenting cells (APCs) from NOD2-deficient mice exhibit increased IL-12p70 synthesis when stimulated by the molecule that give rise to MDP, peptidoglycan, and a well-established Toll-like receptor (TLR) 2 ligand. Furthermore, we showed that addition of MDP to cultures of APCs from NOD2-intact mice led to decreased IL-12p70 responses. ${ }^{5}$ The logical conclusion from these findings was that NOD2 activation by MDP ordinarily results in downmodulation of responses to TLR2 ligands. Finally, we tied this finding to the NOD2 mutation in Crohn's disease by studies showing that transfection of NOD2-deficient APCs with a wildtype NOD2 plasmid led to correction of the IL-12p70 response, whereas transfection of the same cells with a mutated NOD2 plasmid did not lead to such correction.

\section{A MODEL OF COLITIS CAUSED BY NOD2 DEFICIENCY: THE "TWO-HIT THEORY"}

To buttress the concept that NOD2 has regulatory function with respect to TLR2 responses and thus defective NOD2 function leads to excessive TLR2 responses, we first determined if NOD2deficient mice manifest increased susceptibility to induction of E. coli organisms expressing ovalbumin "(ECOVA) colitis." The latter is a colitis caused in mice having $\mathrm{T}$ cells that recognize and mount an inflammation-inducing response to ovalbumin peptide on exposure to recombinant ECOVA organisms expressing this peptide. ${ }^{6}$ Using this model, we showed that NOD2-deficient mice but not NOD2-intact mice administered $\mathrm{T}$ cells that react to ovalbumin peptide develop a transient but intense IL-12-driven colitis when subjected to intrarectal administration of ECOVA. In addition, we showed that mice deficient in both NOD2 and TLR2 are no longer susceptible to ECOVA colitis. Overall, the results obtained from the use of the ECOVA model led to the conclusion that NOD2 mutations lead to colitis because they establish a milieu characterized by an overexuberant "innate" (TLR-2mediated) IL-12p70 response. However, as inflammation does not occur in this model unless a second defect is present, i.e., the presence of $\mathrm{T}$ cells that react to an antigen associated with the intestinal microflora (in the case ovalbumin), the data also suggests that colitis resulting from an NOD2 abnormality requires the presence of a "second hit" to cause disease.

\section{REGULATION OF TLR RESPONSES IN MICE THAT OVEREXPRESS INTACT AND MUTATED NOD2}

The concept that a major function of NOD2 is a negative regulatory function has not been universally accepted, in part because this concept must be reconciled with other studies showing that NOD2 has positive effects on cytokine/chemokine synthesis in under some activation conditions. To throw fresh light on this controversy, we turned to the study of mice expressing increased amounts of NOD2, reasoning that if the negative regulatory function of NOD2 exists, it should be intensified in such mice. ${ }^{7}$

In an initial set of studies we focused on mice that bear a NOD2 transgene under a major histocompatibility complex class II promoter so that within the hematopoietic cell compartment its expression is limited to APCs. We found that APCs from mice bearing the transgene and therefore overexpressing NOD2, but not littermate control mice, mount greatly reduced IL-12p70 responses when stimulated by peptidoglycan. Furthermore, APC responses of the transgenic mice to $\mathrm{Pam}_{3} \mathrm{CysK}_{4}$, a TLR2 ligand that does not contain MDP was equivalent to that in littermate control mice, but such normal responses were subject to much more intense downregulation by addition of MDP to the culture than seen in control mice cultures. Taking these findings to an in vivo arena, we then went on to show that mice bearing a NOD2 transgene were almost totally resistant to the induction of peptidoglycan-induced colitis, an intense and usually fatal colitis occurring in certain mouse strains upon intrarectal instillation of peptidoglycan. Similarly, the transgenic mice developed far less severe 2,4,6-trinitrobenzene sulfonic acid (TNBS)-colitis than their normal littermates when subject to intrarectal instillation of TNBS. Thus, the reduced APC IL-12p70 response to peptidoglycan in vitro was translated to an increased resistance to induced colitis wholly or partially driven by peptidoglycan or TNBS.

In further studies, we evaluated mice expressing increased NOD2 as a result of in vivo administration of plasmids encapsulated in a viral envelope that ensures excellent entry into cells in live mice. In this case, the NOD2 plasmids delivered in this manner gave rise to either a normal (unmutated NOD2) or an NOD2 bearing a mutation similar to that in Crohn's disease. The striking finding here was that the administration of the plasmid encoding intact NOD2 led to complete resistance to the induction of TNBS colitis, whereas the administration of the plasmid encoding a mutated NOD2 had only a minor inhibitory effect on the induction of TNBS colitis.

These studies offer striking confirmation of the view that NOD2 does indeed have negative regulatory function. In addition, they show for the first time that provision of NOD2 can protect a mouse from the development of colitis and may therefore be a novel way of treating Crohn's disease.

\section{TLR RESPONSES INTHE PRESENCE OF INCREASED MDP STIMULATION}

The studies described above showing that overexpression of NOD2 in mice could lead to increased NOD2 negative regulation of the TLR2 response, and protection from colitis led us to consider the possibility that a similar effect could be achieved by increased stimulation of endogenous NOD2 by increased exposure to MDP. In initial studies to explore this possibility, we administered MDP (by intraperitoneal injection) to mice undergoing induction of dextran sulfate sodium (DSS) colitis and TNBS colitis and then monitored the colitis by various parameters. ${ }^{8}$ The result was dramatic in that MDP administration prevented both forms of experimental colitis in mice with intact NOD2 and in the case of DSS colitis had no effect on colitis induced in NOD2-deficient mice. The prevention of colitis 
was evident both at the clinical and histologic level as well as at the level of inflammatory cytokine production. In addition, the MDP-treated mice manifested a reduced NF- $\kappa B$ response and accompanying that the cells exhibited reduced responses when stimulated in vitro with multiple TLR ligands. Thus, for the first time, NOD2 signaling was affected not only the TLR2 as in the NOD2 overexpressions studies, but TLR responses in general.

These in vivo studies suggested that NOD2 signaling might be associated with the generation of one or more factors that affect (downregulate) all TLR responses. Therefore, we conducted in vitro studies in which we prestimulated cells with MDP before stimulation with TLR ligands, reasoning that prestimulation would allow more time for a putative inhibitory factor (IRF) to accumulate and make itself evident. Accordingly, we precultured human monocyte-derived dendritic cells with MDP for $24 \mathrm{~h}$ before we cultured the cells with a broad range of TLR ligands. ${ }^{8}$ The result was quite clear-cut: preincubation with MDP led to lower IL-12p40, IL-10, and IL-6 responses to all of the TLR ligands as compared with the response without preincubation. Of interest, in the case of TLR2 stimulation by peptidoglycan, such preincubation enhanced the inhibiting effect seen with simultaneous incubation with MDP and, even more strikingly, in the case of TLR4 stimulation by lipopolysaccharide or other TLR stimulations, such preincubation reversed enhanced response seen with simultaneous incubation with MDP. In parallel studies of mouse bone marrow-derived dendritic cells, we again observed that preincubation with MDP led to across-the-board reduction in responses as compared with TLR responses alone; however, in this case, no such reduction was observed with cells from mice deficient in NOD2, indicating that the reduced responses were in fact NOD2-specific. Finally, we compared the effect of preincubation with MDP to preincubation with TLR ligands such as LPS. The latter also led to decreased responses, although the pattern and degree of decrease differed somewhat from that obtained with MDP. Thus, the suppressive effect of MDP was part of a general effect of TLR, and NOD-like receptor stimulation and therefore may represent a general mechanism of limiting innate immune responses.

The above in vitro studies led the way to a search for the factor that was responsible for the MDP inhibitory effect. Our approach here was based on the fact that MDP preincubation of cells caused reduced TLR-induced NF- $\kappa \mathrm{B}$ responses, and thus the IRF was likely to be related to this inflammatory pathway. Therefore, we performed extensive western blot analyses of whole cell extracts of cells incubated with MDP to obtain a semiquantitative estimate of the expression of components of the NF- $\kappa B$ pathway in the stimulated cells. ${ }^{8}$ This approach proved successful in that we found that a factor known as IRF4 that had previously been shown to inhibit TLR signaling, was elevated in preincubated cells. To verify that this factor was in fact the inhibitory principle in MDP preincubated cells, we performed gene silencing studies in which we determined the effects of MDP preincubation in cells transfected with siRNA specific for IRF4 (as well as control siRNA). We found that, indeed, cells transfected with IRF4-specific siRNA had a greatly reduced capacity to be inhibited by MDP preincubation. In addition, we showed that a cell line that does not express IRF4 (THP-1 cells) is not subject to inhibition by MDP preincubation unless it is transfected with an IRF4-expressing plasmid. Finally, we performed studies to elucidate the molecular mechanism through which

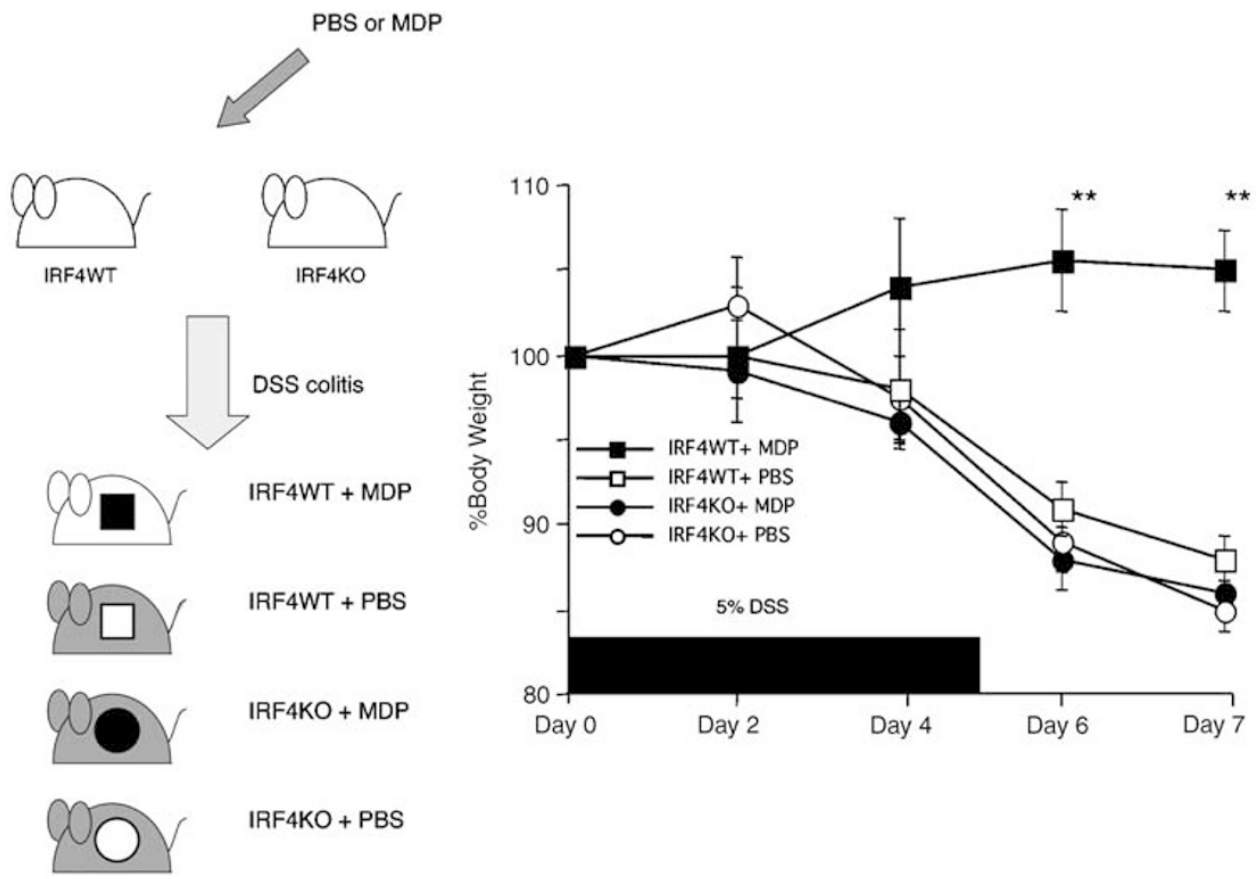

Figure 1 IRF4 signaling is necessary for the suppression induced by MDP. IRF4-intact (IRF4 ${ }^{+/+}$) and IRF4-deficient (IRF4 ${ }^{-/-}$) mice were treated with $5 \%$ DSS in the drinking water for 6 days (days $0-5$ ). On days 0,1 , and 2 , mice were administered MDP or PBS (IP). Body weight changes of IRF4-/or IRF ${ }^{+/+}$mice administered MDP or PBS are shown. ${ }^{* \star} P<0.01$ when compared with PBS-injected IRF4 ${ }^{+/+}$mice. DSS, dextran sulfate sodium; IP, intraperitoneal; IRF4, inhibitory factor; MDP, muramyl dipeptide; PBS, phosphate-buffered saline. 


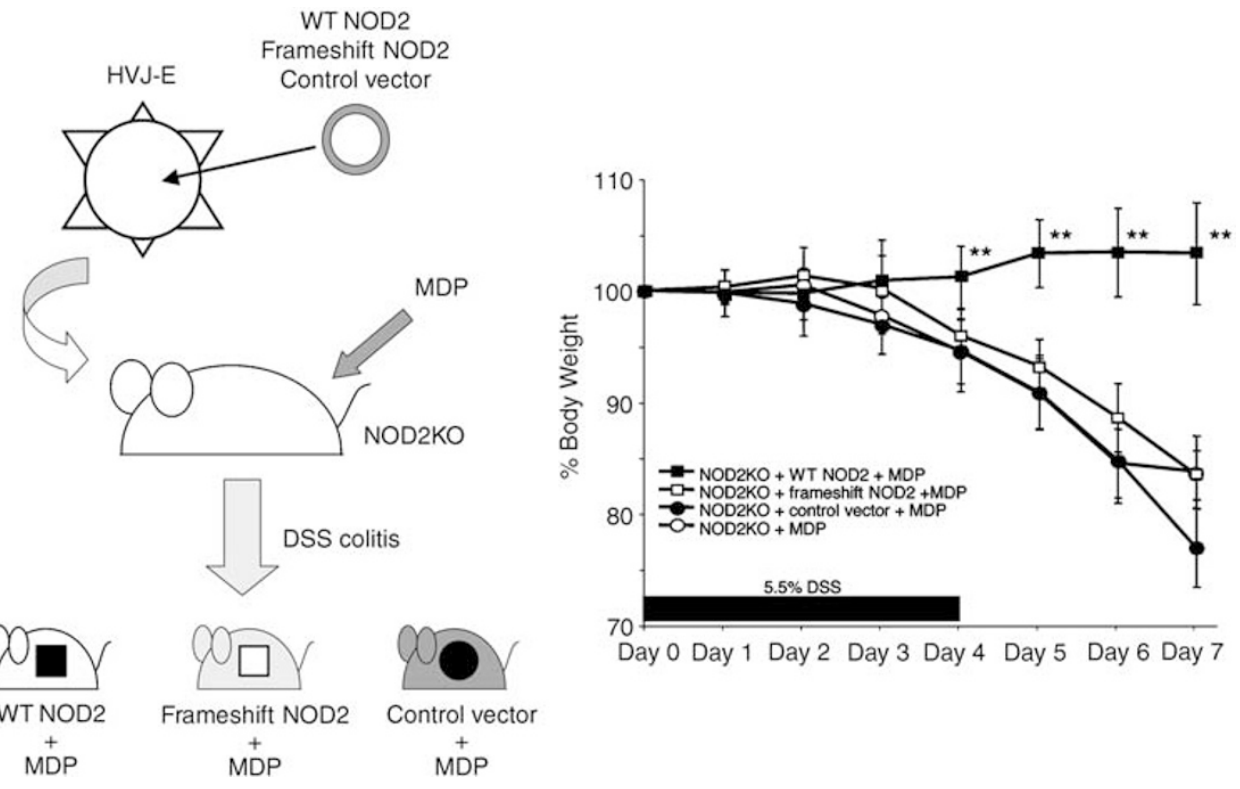

Figure 2 MDP-treated NOD2-deficient mice reconstituted with intact NOD2 are protected from colitis. NOD2-deficient (NOD2 ${ }^{-/-}$) mice were treated with drinking water containing 5.5\% DSS for 6 days (days 0-5). On days 0, 1, and 2, mice were administered MDP and HVJ-encapsulated plasmid (control empty vector, intact or frameshift NOD2). Changes of body weight in MDP administered NOD2-deficient mice reconstituted with intact NOD2, frameshift NOD2, or control empty vector are shown. Body weight changes of MDP administered NOD2-deficient mice given DSS are shown as a control. ${ }^{* \star} P<0.01$ intact NOD2 reconstituted mice compared with control empty vector reconstituted mice. DSS, dextran sulfate sodium; HVJ, hemagglutination virus of Japan; MDP, muramyl dipeptide; NOD, nucleotide oligomerization domain.

IRF4 inhibits TLR responses after MDP preincubation. These studies are as yet incomplete, but at the moment they indicate that IRF4 forms a complex with the NOD2 signaling molecule, receptor interacting caspase-like kinase and this complex then has a negative impact on NF- $\kappa \mathrm{B}$ activation.

At this point we returned to our in vivo models of colitis to determine if IRF4 underlies the ability of MDP to inhibit colitis in vivo. In studies of TNBS colitis, we administered MDP to mice at the time of colitis induction as before, but in this case the mice were treated with siRNA specific for IRF4 to downregulate this factor in vivo. These studies were facilitated by the delivery of the siRNA encapsulated in a viral envelope hemagglutination virus of Japan-envelope that allows efficient in vivo intracellular RNA delivery. ${ }^{8}$ We found that the IRF4-siRNA did downregulate IRF4 expression in the inflamed colon and did prevent the inhibitory effect of MDP administration on TNBS colitis. In a parallel study, we determined the ability of IRF4-deficient mice to respond to MDP administration, in this case to DSS colitis induction. ${ }^{8}$ We found that, although IRF4-deficient mice are normally capable of supporting DSS colitis, MDP administration does not prevent this colitis (Figure 1). Taken together, these studies provide strong verification of the in vitro studies described above demonstrating that MDP inhibition of TLR responses is mediated by IRF4.

In a final series of studies, we sought to relate the above findings to the cause of increased susceptibility to Crohn's disease in patients with NOD2 abnormalities. In these somewhat complex studies, we reconstituted NOD2-deficient mice with plasmids expressing intact NOD2 or a NOD2 bearing a frameshift mutation found in Crohn's disease using the same technique briefly described above, namely administration of plasmid encapsulated in hemagglutination virus of Japanenvelope. We then induced DSS colitis in these mice in the presence and absence of MDP administration. ${ }^{8}$ We found that, intact NOD2 plasmid administration endowed the recipient NOD2-deficient mice with resistance to DSS colitis on MDP administration, whereas frameshift NOD2 had a greatly reduced ability to confer such protection: these mice were poorly protected by MDP administration (Figure 2 ). If we extrapolate this finding to humans with Crohn's disease, we can conclude that NOD2 mutations lead to disease susceptibility because they prevent NOD2 from generating the IRF4 necessary to shut off undue inflammatory responses in the gastrointestinal tract.

\section{SUMMARY}

The studies above provide a body of evidence that a major outcome of NOD2 signaling is the suppression of responses by TLR ligands and thus NOD2 takes its place as an innate immunity responder system with a built-in homeostatic mechanism. However, given the fact that peptidoglycan and its breakdown product MDP is a major constituent of the normal flora in the gut lumen, this homeostatic mechanism assumes great importance as a mediator of gut inflammatory responses and explains the fact that its breakdown in patients with NOD2 mutations leads to susceptibility to Crohn's disease.

\section{DISCLOSURE}

Ivan J. Fuss is a patent holder for the use of anti-IL-12 mAb in the treatment of Crohn's disease. The remaining authors have declared no financial interests.

(C) 2008 Society for Mucosal Immunology 


\section{REFERENCES}

1. Hugot, J.P. et al. Association of NOD2 leucine-rich repeat variants with susceptibility to Crohn's disease. Nature 411, 599-603 (2001).

2. Ogura, Y. et al. A frameshift mutation in NOD2 associated with susceptibility to Crohn's disease. Nature 411, 603-606 (2001).

3. Strober, W., Murray, P.J., Kitani, A. \& Watanabe, T. Signalling pathways and molecular interactions of NOD1 and NOD2. Nat. Rev. Immunol. 6, 9-20 (2006).

4. Abbott, D.W., Wilkins, A., Asara, J.M. \& Cantley, L.C. The Crohn's disease protein, NOD2, requires RIP2 in order to induce ubiquitinylation of a novel site on NEMO. Curr. Biol. 14, 2217-2227 (2004).
5. Watanabe, T., Kitani, A., Murray, P.J. \& Strober, W. NOD2 is a negative regulator of Toll-like receptor 2-mediated Thelper type 1 responses. Nat. Immunol. 5, 800-808 (2004).

6. Watanabe, T., Kitani, A., Murray, P.J., Wakatsuki, Y., Fuss, I.J. \& Strober, W. Nucleotide binding oligomerization domain 2 deficiency leads to dysregulated TLR2 signaling and induction of antigen-specific colitis. Immunity 25, 473-485 (2006).

7. Yang, Z. et al. NOD2 transgenic mice exhibit enhanced MDP-mediated down-regulation of TLR2 responses and resistance to colitis induction. Gastroenterology 133, 1510-1521 (2007)

8. Watanabe, T. et al. Muramyl dipeptide activation of nucleotide binding oligomerization domain 2 protects mice from experimental colitis. J. Clin Invest. 118, 545-559 (2008) 